\title{
SEGURANÇA ALIMENTAR: CONSCIÊNCIA COMEÇA NA INFÂNCIA
}

\author{
A. P. A. GONÇALVES ${ }^{1}$, F. C. LIMA ${ }^{2}$, O. C. P. GASPARETO ${ }^{3}$ \\ 1,2 e ${ }^{3}$ Instituto Federal de Educação, Ciência e Tecnologia do Rio Grande do Norte \\ paulinhagon@msn.com - fabricia_do_carmo@hotmail.com - odisseia.gaspareto@ifrn.edu.br
}

Artigo submetido em janeiro/2011 e aceito em novembro/2011

\section{RESUMO}

Atualmente, a segurança alimentar assume uma preocupação cada vez maior para todos aqueles envolvidos no setor alimentar, pois são diversos os perigos que podem estar contidos na origem de doenças transmitidas por alimentos. E é com essa preocupação que inúmeras entidades, tanto nacionais como internacionais, desenvolvem documentos regularizadores e ações que mobilizem mecanismos de controle. O bem-estar e a saúde associados à alimentação dependem da escolha de alimentos saudáveis e da prática e procedimentos de higiene que minimizem os riscos de contaminação. Tendo como base esses conceitos, este trabalho teve como objetivo levar aos alunos do 60 ano da Escola Estadual Capitão Mor Galvão no município de Currais Novos, noções de higiene e segurança alimentar com o intuito de desenvolver um trabalho de conscientização das crianças quanto à segurança alimentar, já que estas não têm ainda uma opinião formada, fazendo com que sejam disseminados esses novos conhecimentos. A educação de crianças quanto ao tema abordado desenvolverá uma nova percepção em relação ao alimento e a maneira de consumi-lo.

PALAVRAS-CHAVE: segurança alimentar, ensino fundamental, alimentos seguros

\section{JUNIOR GUARDS - FOOD SECURITY: AWARENESS BEGINS AT SCHOOL - Phase 1}

\section{ABSTRACT}

Nowdays, food security assumes an even bigger worry for all those involved in that sector, considering the many dangers that may be contained in the source of food spread diseases. And it's with this worry that many entities, nationals or internationals, develope regulatory documents and actions which mobilizes control mecanisms. The welfare and health associated with feeding depends on the choose of healthy foods and the pract of hygiene proceeds that minimizes the contamination risks. Based on those concepts, this work had as purpose take to the 6th class' students of Capitão Mor-Galvão State School, in Currais Novos city, notions of hygiene and food security, in order to develope a concientization work in those kids about food security, since they don't have a formed opinión yet, dissaminating those new knowledges. The children education about the discussed theme will develope a new perception about food and its ways to consume it.

KEY-WORDS: food security, basic education, safe food. 


\section{SEGURANÇA ALIMENTAR: CONSCIÊNCIA COMEÇA NA INFÂNCIA}

\section{INTRODUÇÃO}

A Segurança Alimentar significa garantir, a todos, condições de acesso a alimentos básicos e de qualidade, em quantidade suficiente, de modo permanente e sem comprometer o acesso a outras necessidades essenciais, com base em práticas alimentares saudáveis, contribuindo, assim, para uma existência digna, em um contexto de desenvolvimento integral da pessoa humana (MENEZES, 1998). A Segurança Alimentar é aplicada ao setor de Alimentação com o objetivo de minimizar ou erradicar as Doenças Transmitidas por Alimentos (DTAs) (III CNSAN, 2007).

Doenças Transmitidas por alimentos (DTA) é um termo genérico, aplicado a uma síndrome, geralmente, constituída de náuseas, vômitos e/ou diarréia. As DTAs são atribuídas à ingestão de alimentos ou água contaminados por bactérias, parasitas, toxinas, príons, agrotóxicos, produtos químicos e metais pesados.

No Brasil, os dados do Sistema de Informações Hospitalares (SIH) do Ministério da Saúde, mostram que de 1999 a 2004, ocorreram 3.410.048 internações por doenças transmitidas por alimentos (DTAs), com uma média de 568.341 casos por ano. As regiões Norte e Nordeste do País são as que apresentam as maiores taxas de incidência, comparadas as outras regiões. A taxa de mortalidade de 1999 a 2004 foi de 25.281 óbitos por DTA, com média de 6.320 óbitos/ano. Os custos com internamento por DTA, de 1999 a 2004 chegam a 280 milhões de reais, com média de 46 milhões de reias por ano. E que, na maioria das vezes os casos de surtos alimentares acontecem em casa $(48,5 \%)$, seguidas de restaurantes $(18,8 \%)$ e escolas $(11,6 \%)$, mas também é interessante ressaltar que na maior parte dos casos não há a determinação de onde ocorreu a contaminação. (SECRETARIA DE VIGILÂNCIA SANITARIA, 2005)

Uma parcela da população brasileira não tem acesso às informações referentes a um alimento seguro. Hoje, a divulgação dos alimentos seguros está sendo feita em grandes supermercados, em trabalhos nas comunidades e nas escolas.

Quanto mais cedo o indivíduo tiver conhecimento do que é Segurança Alimentar, maior será a probabilidade de se influir favoravelmente na formação de hábitos desejáveis. Fazendo com que os alunos analisem as informações, as assimilem e formem suas próprias opiniões sobre o assunto. Como formadores de opinião, as crianças levarão as discussões e aprendizados para suas casas e comunidades, disseminando um novo conceito e extinguindo antigos tabus com relação ao preparo e armazenamento de alimentos.

A escola é o espaço ideal para o desenvolvimento de conhecimentos, atitudes e habilidades. Portanto este estudo teve como objetivo levar as Escolas Públicas os conhecimentos básicos da Higiene e Segurança Alimentar para as crianças do 60 ano, na faixa etária de 9 a 11 anos, do município de Currais Novos, RN. 


\section{MATERIAIS E MÉTODOS}

O estudo foi desenvolvido por alunos do Instituto Federal Do Rio Grande do Norte, Campus Currais Novos do Curso Técnico em Alimentos. Para que os mesmos fossem multiplicadores da Segurança Alimentar, os alunos passaram por uma capacitação, que envolviam os mais variados temas, que são relacionados a seguir:

- Tipos de Perigos nos Alimentos (biológicos, físicos e químicos)

- Doenças Transmitidas por Alimentos

- Noções de Higiene Pessoal (pessoal, na manipulação, ambiente)

- Legislações

- Como escolher um alimento saudável

A escola participante não foi escolhida ao acaso, mas no ano de 2010 o Campus Currais Novos adotou a Escola Estadual Capitão Mor Galvão, através do Projeto Adote uma Escola, que Consiste em melhorar a estrutura de Escolas públicas da comunidade, então optou-se por desenvolver a primeira fase do projeto lá.

O público alvo do projeto foram os alunos do 60 ano da referida escola. Eles receberam orientações quanto à higiene pessoal, segurança alimentar, perigos que podem estar contidos nos alimentos, como identificar os alimentos possíveis de causar doenças, assim como manipular os alimentos com higiene.

Como recursos didáticos utilizou-se palestras com equipamentos de multimídia, brincadeiras, vídeos e aplicação de questionários. Que foram realizados, em sua totalidade, na escola, pois foi cedida uma hora por semana para a realização dessa atividade, junto às crianças.

\section{RESULTADOS E DISCUSSÕES}

As palestras foram ministradas inicialmente, como uma conversa onde foi explicada a intenção do projeto, em seguida, surgiram discussões sobre higiene, higienização dos alimentos, micróbios (microrganismos), entre outros questionamentos. A partir deste princípio, começou-se a avaliar o nível de conhecimento dos alunos sobre o tema abordado.

Foram aplicados questionários sobre conhecimentos básicos de higiene e procedimentos que se deve ter para evitar a contaminação dos alimentos e assim consumir um produto livre de contaminantes, constatando um resultado positivo para a maioria (Figura 1). Em seguida, foram discutidos os erros e apontadas às ações corretas. Quanto ao quesito Higiene Pessoal, apresentou-se vídeos infantis deixando explícito o porquê de uma higiene adequada.

Com relação à Segurança Alimentar, foi mostrada a cadeia produtiva dos alimentos desde o plantio ou criação, até a chegada à mesa do consumidor, além de como escolher um alimento seguro. A maioria dos alunos fizeram relatos de situações passadas, mostrando grande interesse sobre o 
assunto. Para fixar conteúdo, aplicou-se atividade (resumo do que foi discutido) sobre os temas abordados anteriormente, obtendo bons resultados (Figura 2), pois todos mostraram ter compreendido a importância de ter uma boa higiene, tanto pessoal como alimentar e de que forma podemos contribuir para garantir a qualidade e assegurar que os alimentos sejam isentos de contaminações.

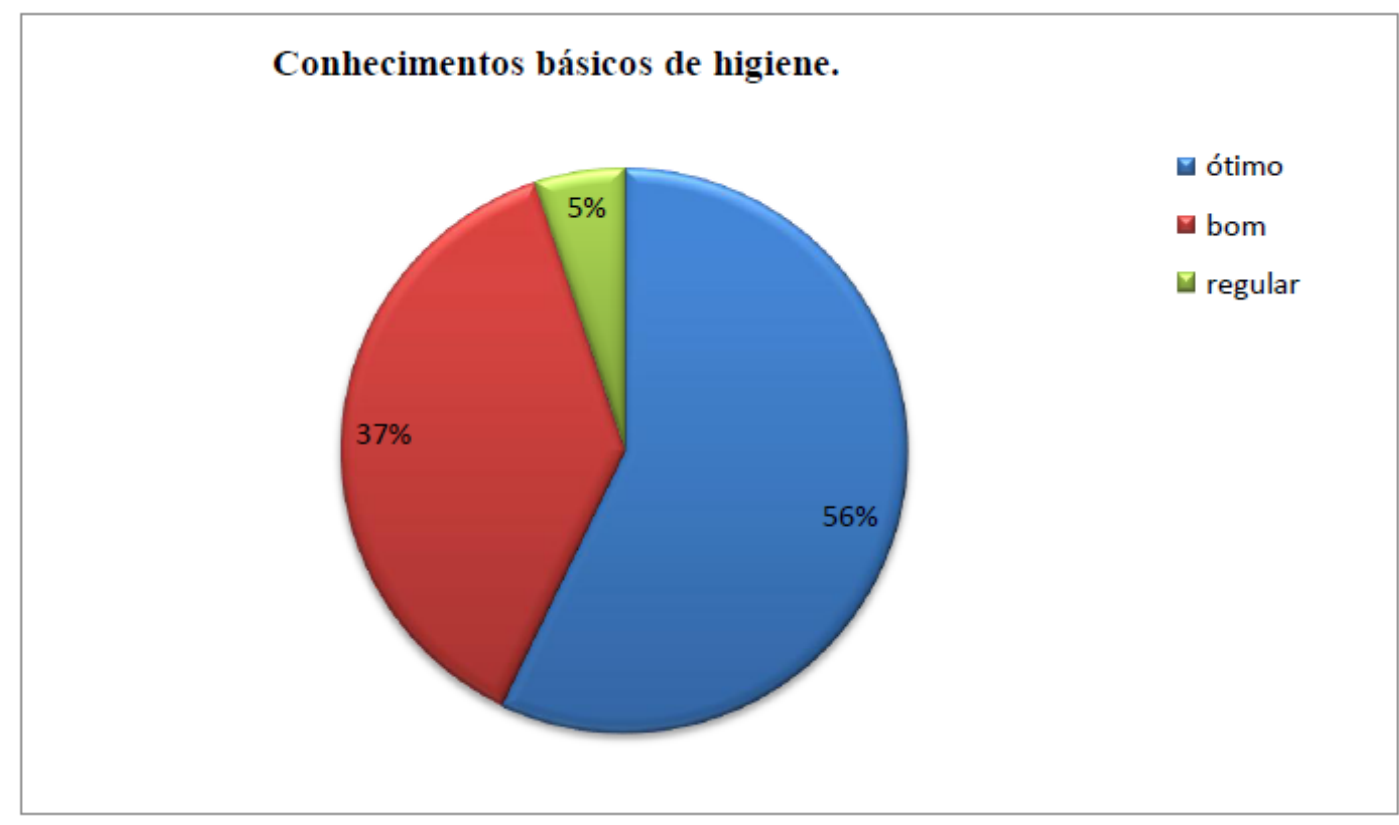

Figura 1 - Questionário avaliativo sobre o tema Higiene.

Atividade sobre a compreenção da cadeia produtiva e cuidados com os alimentos na mesma.

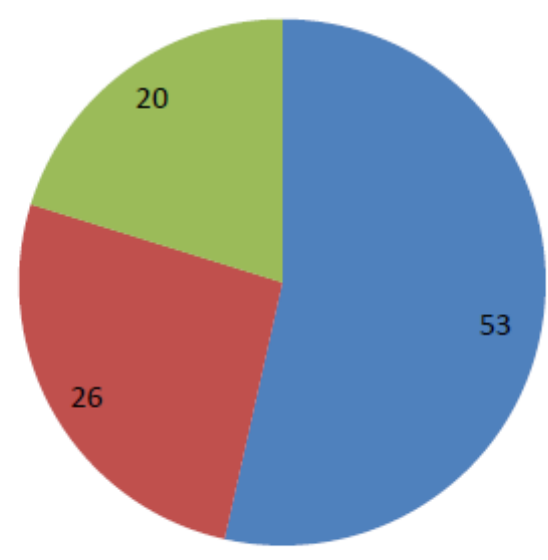

Figura 2 - Gráfico dos resultados obtidos com a atividade sobre a cadeia produtiva dos alimentos e o cuidado com a mesma. 
Quanto aos Perigos Físicos, Químicos e Biológicos, cada um foi caracterizado, exemplificado e mostrado os cuidados que se deve ter para que estes não venham a comprometer a saúde e integridade do consumidor. Surgiram diversas dúvidas em relação a este assunto, então foi aplicado questionário sobre o exposto (do tipo perguntas e respostas, identificarem em figura erros, relacionar imagens a frases, marcar alternativas corretas), onde foi comprovado que a diferenciação de cada perigo não foi compreendida completamente.

Na Figura 3 pode-se observar que apesar de $59 \%$ da turma ter compreendido, obteve-se $40 \%$ de entendimento regular, o que identifica um fraco entendimento do assunto.

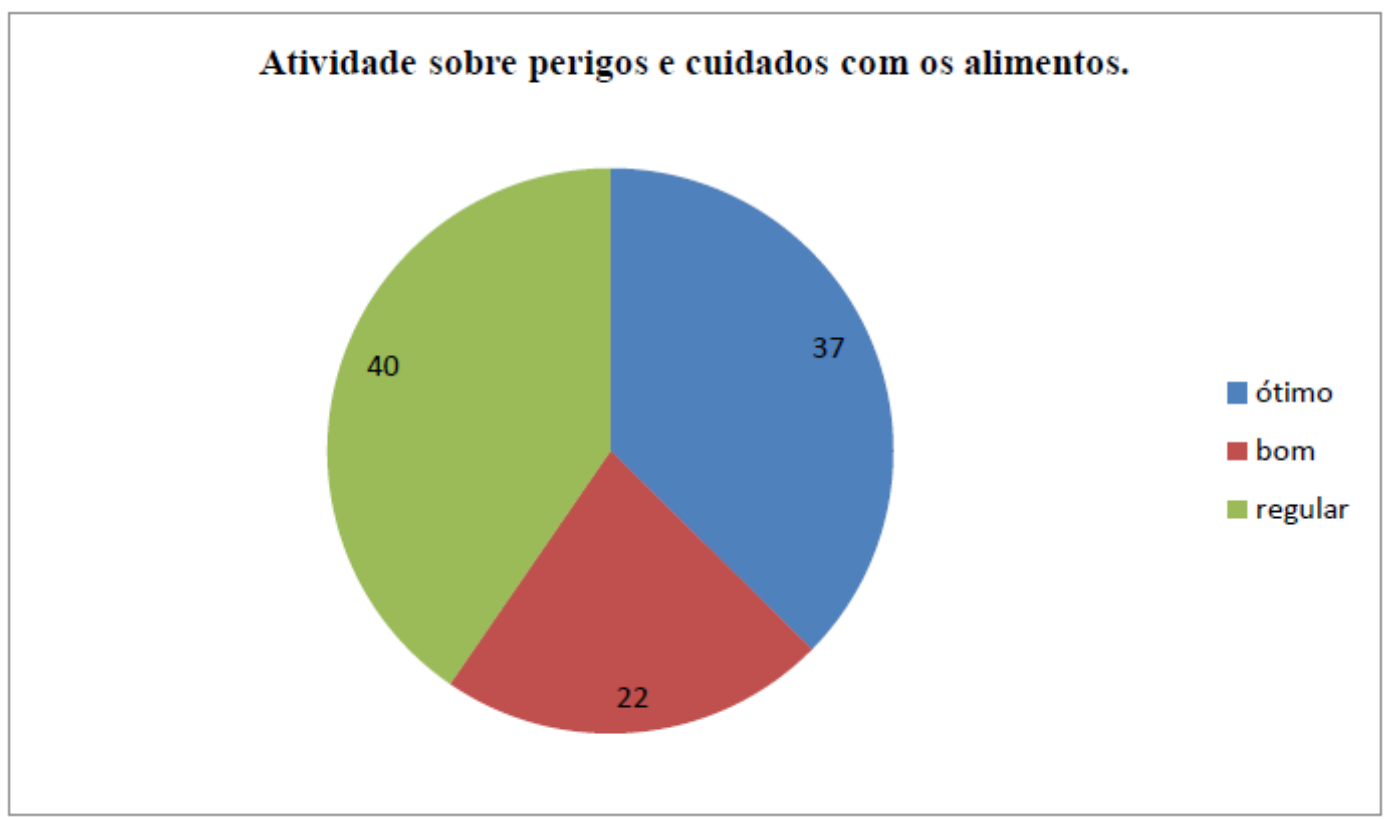

Figura 3 - Gráfico dos resultados do nível de compreensão dos alunos sobre o tema os perigos alimentares.

Para se ter uma resposta da turma quanto aos temas abordados até então, foram introduzidas brincadeiras de perguntas e respostas, onde enfatizou-se os tipos de perigos que podem contaminar os alimentos. A dinâmica não mostrou resultados significantes, pois a maioria dos participantes não sabia responder às perguntas (cerca de $80 \%$ ), as quais já haviam sido discutidas em aulas passadas. A falta de interesse dos alunos pela brincadeira deve ter se dado pelo fato dos brindes, serem entregues a todas as crianças e não só a que acertasse as questões.

\section{CONCLUSÕES}

Após as palestras realizadas, com o emprego de brincadeiras e atividades para avaliar o nível de aprendizagem, chegou-se à conclusão de que a turma dominou com facilidade os assuntos relacionados aos conhecimentos básicos de higiene e os cuidados que se deve ter com os alimentos na cadeia produtiva, para assim obter um alimento seguro sem contaminações. $O$ conteúdo referente a 
perigos nos alimentos não foi assimilado, tendo em vista os valores encontrados, nos quais o percentual de ótimo reduziu em relação aos outros e o de regular aumentou.

\section{AGRADECIMENTOS}

Agradecemos ao IFRN, Campus Currais Novos e a Escola Estadual Capitão Mor Galvão por possibilitar o desenvolvimento desse trabalho.

\section{REFERÊNCIAS BIBLIOGRÁFICAS}

1. MENEZES, F. Panorama Atual da Segurança Alimentar no Brasil, 1998. Disponível em: http://pagesperso-orange.fr/amar-bresil/documents/secual/san.html > Acessado em 19/04/2010.

2. RELATÓRIO FINAL. III Conferência Nacional de Segurança Alimentar e Nutricional. Por um desenvolvimento sustentável com soberania e Segurança Alimentar e Nutricional. 2007 Disponível em: <http://www.fomezero.gov.br/documentos/>. Acessado em 19/04/2010.

3. SECRETARIA DE VIGILÂNCIA EM SAUDE. Boletim Eletrônico Epidemiológico. Vigilância Epidemiológica das Doenças Transmitidas por Alimentos no Brasil, 1999 - 2004. Ano 5, no6, dez. 2005. 